\title{
I8. COMMISSION DES LONGITUDES PAR TÉLÉGRAPHIE SANS FIL
}

Président: Général G. Perrier.

Secrétatre: M. A. Lambert, Observatoire de Paris.

Membres: MM. Bianchi, Bowie, Bowyer, Carty, Coculesco, Da Costa Lobo, Deslandres, Esclangon, Fayet, Fichot, Hashimoto, Iwanoff, Jouaust, E. Kohlschütter, Krassowski, Lejay, Littell, Moreau, Niethammer, Nörlund, Nušl, Sampson, Stewart, Volta.

Les premiers mots de ce Rapport seront un souvenir ému à la mémoire de notre regretté Président, le Général Ferrié, brutalement arraché, en pleine activité physique et intellectuelle, au respect et à l'affection de tous, le 16 février 1932. Il fut l'instigateur et l'animateur des travaux auxquels se consacre notre Commission. Nous ne saurions lui rendre de plus bel hommage-celui-là même qu'il eut souhaité sans doute-qu'en nous attachant à poursuivre, dans la voie qu'il avait tracée en I926 et qu'il avait commencé de prolonger en vue des opérations de I933, la mise au point d'une entreprise de haute portée scientifique et qui était au tout premier plan de ses préoccupations.

Deux résolutions furent adoptées par notre Commission lors de la $3^{\mathrm{e}}$ Assemblée générale de l'U.A.I. tenue à Leyde en juillet 1928 .

La première exprimait le désir de voir publier un fascicule d'ensemble où seraient consignées les valeurs définitivement adoptées pour les longitudes des stations qui ont pris part aux opérations d'octobre-novembre I926; les caractéristiques des méthodes et des instruments employés y étant succinctement indiquées.

Un résumé, rédigé par les soins de votre Secrétaire a pu être imprimé en I929 grâce à une subvention accordée par le "Conseil International de Recherches." Il a été largement distribué. C'est une brochure de 48 pages avec une carte, intitulée La Revision des Longitudes mondiales (octobre-novembre 1926).

La deuxième résolution s'exprimait ainsi:

L'opération des longitudes sous la forme qui lui a été donnée en 1926 doit être reprise. La date fixée pour son renouvellement est, en principe, l'année qui suivra la prochaine réunion de l'Union Astronomique. Son époque exacte et son programme seront déterminés par la Commission au cours de cette réunion de l'Union. D'ici là, les observatoires sont invités à poursuivre d'une façon à peu près permanente la détermination de la longitude en portant leur attention sur l'étude des erreurs systématiques que les travaux de I926 ont pu révéler: instantanéité de l'enregistrement, qualité des niveaux, temps perdu de la vis micrométrique et équation personnelle absolue des observateurs, etc. Tous les renseignements ainsi recueillis seront portés à la connaissance de la Commission avant sa prochaine réunion.

En conséquence, le 8 janvier I930, le Président de la Commission mixte des Longitudes par T.S.F. adressa à toutes les personnalités et organismes intéressés une première circulaire proposant l'établissement, en I933, de trois circuits fondamentaux:

I. Greenwich-Tokio-Vancouver-Ottawa (déjà déterminé en 1926).

II. Alger-Zi Ka Wei-San-Diego ou Mount-Hamilton-Washington (déjà déterminé en $\mathrm{I926)}$.

III. Le Cap-Adelaide-Rio de Janeiro (nouveau).

De plus, de nombreuses opérations secondaires pourraient être faites.

La deuxième circulaire adressée le I8 avril I930 à plus de $35^{\circ}$ destinataires

S A U IV 
faisait état des réponses à la première. Les points suivants pouvaient y être provisoirement fixés par le Président de la Commission:

“(I) Le succès des opérations de l'entreprise d'octobre-novembre I926 conduit à adopter un plan d'ensemble analogue à celui qui fut alors suivi. On ajoutera néanmoins un troisième circuit fondamental dans l'hémisphère sud, en sorte que les trois polygones circumterrestres envisagés sont:

(a) Greenwich-Tokio-Vancouver-Ottawa.

(b) Alger-Zi Ka Wei-San-Diego.

(c) Le Cap-Adelaide (ou tout autre station d'Australie)-Rio de Janeiro.

"D'autre part, les stations fondamentales de Paris et Washington, ainsi que le plus grand nombre possible d'autres points géographiques devront être également rattachés aux sommets des polygones circumterrestres.

"(2) Les observations s'effectueront en principe en octobre-novembre I933; il y a en effet intérêt, pour déceler les variations possibles de longitude tout en s'affranchissant des erreurs saisonnières qui peuvent affecter les mesures, à effectuer les opérations aux mêmes mois qu'en I926.

“(3) Les observations astronomiques se feront, autant que possible au moyen de petits instruments méridiens à micromètre impersonnel muni, s'il se peut, d'entraînement mécanique du fil mobile. Il serait souhaitable d'adjoindre à l'instrument méridien un astrolabe à prisme, sous la condition que celui-ci fût pourvu d'un appareil de mesure d'équation personnelle absolue.

"A défaut d'astrolabe, tout Observatoire possédant deux instruments méridiens d'équipement moderne aurait intérêt à les faire concourir simultanément aux mesures.

“(4) Les émissions de signaux radio-électriques seront faites sur ondes longues et sur ondes courtes.

"Il est recommandé aux stations de longitude de s'outiller de façon à enregistrer sur chronographe les signaux radio-électriques. Le rythme des émissions sera conservé toutefois, de manière à permettre l'emploi de la méthode des coïncidences.

“Je m'efforcerai d'obtenir que certains postes commerciaux ou officiels consentent à transmettre des séries spéciales de signaux horaires, le nombre des émissions de ces signaux faites en temps normal paraissant insuffisant.

"La marche des pendules modernes à pression et températures constantes se déterminant avec sécurité, la nécessité d'observations astronomiques et d'émissions radios très voisines dans le temps ne s'impose plus. Ce qu'il faut tâcher de réaliser en chaque station ce sont les conditions optima de réception des signaux radios."

Chaque destinataire était invité à faire connaître ses possibilités, ses intentions, ses désidérata.

Les avis et les suggestions formulés permirent l'établissement d'un programme de questions à examiner par la Commission mixte des Longitudes, lors de l'Assemblée Générale de l'Union géodésique et géophysique internationale tenue à Stockholm en août I930. Le Bureau de la Commission rédigea un rapport augmenté de pièces annexes, qui servit de base à la discussion. Les conclusions de la Commission furent communiquées le 24 septembre I930, dans une troisième circulaire. Ces conclusions condensées en deux vœux qui reçurent l'approbation de l'Union, étaient: 


\section{Premier Vceu}

"Des mesures renouvelées et précises des différences de longitude entre des points du globe convenablement choisis devant permettre en particulier de fixer la valeur de certaines hypothèses concernant une déformation de la surface de la terre, qu'il s'agisse de la dérive des continents de $\mathbf{M}$. Wegener ou des plissements sous-marins le long des côtes soupçonnés par M. Vening Meinesz.

"L'Union géodésique et géophysique internationale émet le vœu que, pendant la deuxième opération mondiale de longitudes qui aura lieu à l'automne de I933, des stations conjuguées, parfaitement outillées au point de vue des observations astronomiques et des réceptions radio-télégraphiques, fonctionnent dans les régions suivantes:

(I) Pointe du Groënland et Écosse.

(2) Madagascar et Mozambique.

(3) Ile de Ternate et Ménado (Indes Néerlandaises).

"L'Union souhaite obtenir l'approbation et le concours des gouvernements et organismes de qui dépend la réalisation de ce vœu."

\section{Deuxième Væu}

"L'opération mondiale des longitudes de I926 avait, dans la pensée de ses promoteurs, le caractère d'un essai devant permettre de fixer les possibilités et les méthodes. Sa réussite et la qualité des résultats, qui ont généralement dépassé les espérances, ont fait néanmoins regretter une fois les calculs achevés, qu'une étude d'ensemble comparative et critique n'ait pas été prévue. Celle-ci n'a pu, faute de moyens matériels, être réalisée jusqu’ici, et il conviendra de l'entreprendre dès que les circonstances le permettront.

"Il sera en tous cas indispensable de faire une étude critique semblable pour ce qui concerne les opérations de I933.

“De plus, ce travail doit être complété par l'examen des problèmes que soulèvent les observations quotidiennes des signaux horaires dans les grands Observatoires et les variations des différences de longitudes qui en résultent.

"Le Bureau international de l'Heure, étant un organisme dont l'activité est intimement liée à ces questions, apparaît à l'Union géodésique et géophysique internationale comme étant tout spécialement désigné pour assurer l'ensemble de ces travaux, qui ne sera que le prolongement de son rôle actuel.

“ Reconnaissant qu'on lui demande ainsi d'assumer une charge nouvelle à laquelle ses ressources matérielles actuelles ne lui permettent pas de faire face, reconnaissant, de plus, les services signalés que le Bureau international de l'Heure rend d'une façon permanente aux géodésiens, l'Union géodésique et géophysique internationale a le désir, sitôt que l'état amélioré de ses finances le lui permettra, d'accorder une subvention annuelle au Bureau international de l'Heure, pour le mettre en mesure d'étendre dans le sens indiqué le champ de son activité."

Afin d'assurer en toutes les régions du globe des réceptions convenables de signaux horaires, tant sur ondes longues que sur ondes courtes, il pourra être nécessaire d'ajouter quelques émissions supplémentaires aux signaux horaires régulièrement radio-diffusés. A cet effet, le Président écrivit le 20 mai I93I à Tananarive, au Cap de Bonne Espérance, à Lembang, à Tokio, à Buenos Aires, à Hanoï. Les réponses définitives des divers organismes directeurs ne nous sont pas parvenues encore. 
La circulaire No. 4 en date du 31 juillet I93I, comportait: (I) une carte des stations astronomiques et radio-télégraphiques devant vraisemblablement prendre part aux opérations de I933; (2) un relevé des émissions horaires régulières sur ondes longues; (3) une note de M. Driencourt sur l'équation personnelle dans les observations à l'astrolabe à prisme; (4) un article de M. Esclangon sur l'A mélioration des observations méridiennes; (5) une note de M. Lambert sur l'équation personnelle dans les observations de passages; (6) une note de M. Jouaust sur les retards dans les enregistrements des signaux horaires.

Enfin, la cinquième et dernière circulaire que signa le Général Ferrié, expédiée le 29 décembre I93I, proposait une répartition des signaux horaires à ondes longues à enregistrer ou écouter, suivant la situation géographique des Observatoires ou stations astronomiques.

A la date du $I^{\text {ex }}$ juin I932, quatre-vingt-cinq Observatoires permanents ou stations astronomiques ont fait connaître leur intention de prendre part aux opérations de I933. Pour un petit nombre d'entre eux l'adhésion n'est pas définitive, mais il faut envisager par contre une collaboration de stations nouvelles, en Sibérie notamment, qui ne sont pas portées sur la carte jointe au présent Rapport.

Les cinq Observatoires de l'Amérique du Sud qui ont déjà promis leur concours à l'opération de I933 confirmeront sans doute l'erreur qui semble affecter les longitudes des points fondamentaux de cette région déterminées jusqu'à ces dernières années.

Les positions admises découlaient des mesures effectuées en I878-I879 par les Officiers de la Marine des États-Unis. L'Opération des Longitudes mondiales d'octobre-novembre I926, ainsi que des déterminations ultérieures en 1927 et x928, ont révélé un écart systématique de l'ordre de I seconde.

On aurait ainsi les valeurs approchées suivantes:

\begin{tabular}{|c|c|}
\hline \multicolumn{2}{|c|}{ Longitudes anciennes moin } \\
\hline $\mathrm{La} \mathrm{Paz}$ & $-\mathbf{I}, \mathbf{2}^{\mathrm{B}}$ \\
\hline Santiago de Chili & $-I, I$ \\
\hline La Plata & $-I, I$ \\
\hline Rio de Janeiro & $-0,6$ \\
\hline Buenos Aires & $-0,5$ \\
\hline
\end{tabular}

Répondant au premier vœu exprimé par l'Assemblée de Stockholm, l'Institut géodésique du Danemark est disposé à établir une station astronomique à Kornok (Groënland). Le Portugal fera exécuter des observations à Lourenço-Marquez. Mais les autorités des Indes Néerlandaises n'ont pas fait connaître encore que des Missions s'installeraient à Ternate et Ménado.

La Direction du Bureau international de l'Heure, pour satisfaire au second vœu, soumettra à l'approbation de la Commission de l'Heure de l'U.A.I. au cours de la présente Assemblée un programme d'exécution en harmonie avec ce vœu.

Compte tenu des avis reçus, l'examen du programme suivant est proposé à l'attention de la Commission, en vue de l'Opération des Longitudes mondiales de r933:

\section{(I) Buts de l'Opération}

L'enregistrement continu, tout au long de l'année, en différents Observatoires du globe, des mêmes signaux horaires régulièrement émis par les grandes stations radio-télégraphiques constitue évidemment la vraie solution du problème des longitudes; ainsi peuvent se relier quelques Observatoires importants, bien dotés 
en personnel et matériel, suivre en plus les variations saisonnières de leurs différences de longitude, et en tirer des conclusions. Une semblable synthèse est exécutée depuis Ig29 au Bureau international de l'Heure pour neuf points du globe. Mais la plus grande majorité des Observatoires n'est pas outillée pour réaliser un tel programme; toutefois il leur est possible d'y adhérer durant un temps limité, deux mois par exemple. De plus, les différentes nations peuvent avoir le désir de profiter d'une organisation d'ensemble pour fixer les positions de quelques points secondaires de leurs territoires. Enfin, l'un des buts de l'opération étant d'éprouver la fixité des continents, c'est en des points du globe qui ne coïncident pas avec la situation des grands Observatoires que le contrôle des hypothèses de Wegener peut se faire en un laps de temps relativement court. Ces raisons, dont quelques-unes ont conseillé l'entreprise de I926, demeurent valables pour I933.

\section{(2) Circuits fondamentaux}

Trois circuits principaux sont prévus:

(a) Greenwich-Tokio-Vancouver-Ottawa.

(b) Alger-Zi Ka Wei-San-Diego.

(c) Le Cap-Adelaide-Wellington-Buenos Aires.

(Il a été possible à cette dernière station de s'équiper d'une façon toute moderne, en ces derniers mois.)

Les Observatoires fondamentaux de Paris et de Washington ainsi que le plus grand nombre possible d'autres stations devront être rattachés aux sommets des circuits.

\section{(3) Date des Opérations}

Les opérations commenceront le $I^{\text {er }}$ octobre 1933 et prendront fin le 30 novembre I933; il est indispensable, en effet, pour l'élimination de l'erreur saisonnière dans les comparaisons qui seront faites avec les résultats de I926, d'observer à la même époque que lors de la précédente entreprise. Toutefois, comme les conditions atmosphériques sont alors défavorables en certaines stations qui auront avantage à devancer un peu ou à retarder le début des opérations, on invite les grands Observatoires à vouloir bien exécuter leur programme du I5 septembre au I5 décembre I933.

\section{(4) Observations méridiennes}

Il est recommandé de faire usage de lunettes de passage munies de micromètres auto-enregistreurs. Chaque Observatoire possédant plusieurs instruments de ce genre est instamment sollicité de les consacrer aux déterminations d'heure durant l'opération des longitudes. L'éviction probable des erreurs systématiques se reconnaît le mieux à l'accord de deux instruments différents: c'est un contrôle auquel chacun est invité à se livrer s'il le peut avant l'automne I933. (A Paris, par exemple, l'écart moyen entre les corrections de pendule fournies par l'instrument de passage de $8 \mathrm{~cm}$. d'ouverture et le cercle méridien de $\mathrm{I} 9 \mathrm{~cm}$. d'ouverture a été de $-0,003^{\mathrm{s}}$ pour une moyenne de quinze soirées communes, avec une erreur probable de $\pm 0,0065^{\mathrm{s}}$.)

Malgré l'exiguïté de l'équation personnelle dans l'emploi du micromètre autoenregistreur, il est bon que les observateurs puissent la mesurer au moyen d'un appareil spécial, du genre de ceux qui existent notamment à l'Observatoire Naval de Washington, et à l'Observatoire de Paris. A tout le moins, il est recommandé de faire usage du prisme à réversion. 


\section{(5) Observations faites à d'autres Instruments}

D'autres instruments, appliquant la méthode des hauteurs égales, seront peutêtre mis en service. Si leur construction ne leur assure pas un caractère d'impersonnalité, l'emploi de tels instruments ne présentera d'intérêt que s'ils sont accompagnés d'un appareil propre à mesurer l'équation personnelle de l'observateur.

\section{(6) Choix des Etoiles}

L'homogénéité des catalogues modernes n'est pas assurée. Si l'on compare les différences d'ascensions droites entre les étoiles du système d'Auwers et celles du système d'Eichelberger dans la zone de déclinaison voisine de $50^{\circ}$, on constate à douze heures d'intervalle des écarts atteignant en moyenne $0^{5}, 03$. Il vaudra donc mieux pour les observations méridiennes faites à latitudes voisines opérer aux mêmes heures locales, c'est à dire observer sensiblement les mêmes groupes d'étoiles. La conservation de l'heure pendant une courte durée, la sécurité de la marche des pendules maintenues à température et sous pression constantes sont assurées actuellement avec une très haute précision. Les réceptions de signaux horaires peuvent sans inconvénient se faire à un moment éloigné de celui des observations astronomiques.

\section{(7) Méthodes d'Observation et de Réduction}

La méthode la plus généralement suivie pour la détermination astronomique de l'heure aux instruments méridiens, consiste à observer des étoiles symétriquement disposées au nord et au sud du zénith et en son voisinage. L'observation des polaires n'est pas indispensable; de la sorte, l'équation personnelle dite de bissection est pratiquement éliminée, ainsi que l'influence de l'azimut instrumental. Mais une détermination très sure de l'inclinaison de l'axe est indispensable: on ne saurait trop recommander les différentes vérifications: usage de deux fioles pour les niveaux, contrôle par l'horizon à mercure. Le temps perdu de la vis devra être mesuré avec soin.

Afin de faciliter la tâche de l'Office qui vraisemblablement centralisera et discutera l'ensemble des mesures, il serait désirable d'uniformiser la présentation et les notations des résultats bruts. Cela s'applique aussi bien aux observations astronomiques qu'aux enregistrements de signaux horaires; on trouvera aux Annexes une proposition de feuille-type à remplir par chaque station et à adresser à l'Office centralisateur.

\section{(8) Choix des Stations radio-télégraphiques}

Il a été reconnu qu'il valait mieux réduire le nombre des émissions horaires quotidiennes effectuées en 1926, en les limitant aux émissions régulières des postes puissants de grande portée. Toutefois, il est apparu utile de demander à Honolulu, à Malabar et à Saiggon une émission supplémentaire chaque jour pendant la période des observations. Les adhésions des deux premiers postes n'ont pas encore été reçues. La circulaire datée de décembre I93I indique les Signaux horaires à ondes longues à enregistrer ou à écouter dans les diverses régions du globe. C'est un schéma sur lequel la Commission aura à se prononcer.

Les incertitudes qui pèsent sur les réceptions des ondes courtes doivent laisser toute liberté à ceux qui ne sont pas équipés pour recevoir les ondes longues; aucun choix ne peut être préconisé à leur égard. Comme vraisemblablement tous les signaux sur ondes courtes, susceptibles d'être enregistrés au Bureau international de l'Heure, le seront en octobre-novembre I933, qu'un certain nombre d'entre eux 
seront également enregistrés dans les grands Observatoires, le rattachement des stations astronomiques qui les auront utilisées à l'exclusion des autres signaux, sera possible.

(9) Mesure des Retards à l'Enregistrement

La mesure de cette erreur systematique est de la plus haute importance; les deux Notes jointes de M. R. Jouaust, qui a particulièrement étudié le phénomène, éclairent la question. Le procédé simple d'évaluation des retards qu'il préconise sera d'une application aisée, si les stations radio-télégraphiques veulent bien faire suivre les émissions horaires d'un trait long, d'une durée de dix secondes par exemple. Il n'est pas question, bien entendu, de modifier les pendulettes émettrices; le signal peut être commandé par un manipulateur et commencerait 5 secondes après le début du dernier signal horaire.

\section{(Io) Discussion et Publication des Résultats}

Sous réserve de l'approbation de la Commission de l'Heure, le Bureau international de l'Heure, ainsi qu'il a été dit précédemment, est tout disposé à répondre au vœu exprimé à Stockholm et à entreprendre une discussion d'ensemble des résultats, si les moyens matériels lui en sont fournis. Ayant centralisé les observations effectuées aux différentes stations, le Bureau ferait paraître d'abord un fascicule fournissant les heures définitives des différentes émissions aussi promptement que possible. L'impression du Mémoire comportant une discussion critique d'ensemble serait préparée ensuite. Une aide financière des différents organismes intéressés à l'entreprise devra être envisagée, à tout le moins pour ce dernier objet, fort onéreux.

Général G. PERrier

Président de la Commission

A. LAMBERT

Secrétaire de la Commission 